\title{
Correction to: Probing a Two-Way Parallel T\&I Corpus for the Lexical Choicesof Translators and Interpreters
}

Oi Yee Kwong

\section{Correction to:}

Chapter "Probing a Two-Way Parallel T\&I Corpus for the Lexical Choices of Translators and Interpreters"

in: V. X. Wang et al. (eds.),

New Perspectives on Corpus Translation Studies,

New Frontiers in Translation Studies, https://doi.org/10.1007/978-981-16-4918-9_5

The original version of the book was published with incorrect spell error in the abstract section, now corrections has been incorporated. The chapter and book have been updated with the changes. 